\title{
Health-related quality of life impact of a triple combination of olmesartan medoxomil, amlodipine besylate and hydrochlorotiazide in subjects with hypertension
}

\author{
Pedro Marques da Silva', Uwe Haag ${ }^{2}$, Julian F Guest ${ }^{3,4}$, John E Brazier ${ }^{5}$ and Marco Soro ${ }^{6 *}$
}

\begin{abstract}
Background: A post-hoc analysis was performed on the data from a 54 weeks phase III study (ClinicalTrials.gov identifier: NCT00923091) to measure changes in the health-related quality of life (HRQoL) of 2,690 patients aged $\geq 18$ with moderate-to-severe hypertension who received one of six doses of olmesartan/amlodipine/hydrochlorothiazide (OLM) AML/HCTZ), using the MINICHAL and EQ-5D instruments.

Methods: Descriptive statistics were used to assess blood pressure and HRQoL scores over the study period. Analysis of covariance (ANCOVA) was used to identify those factors that could possibly have influenced HRQoL. Linear regression was used to assess the relationship between changes in blood pressure and HRQOL scores.

Results: Patients' baseline MINICHAL mood and somatic domains scores were 5.5 and 2.6. Over the study period HRQoL improved as both MINICHAL scores decreased by 31-33\%. Patients' baseline EQ-5D index and VAS scores were 0.9 and 73.4 respectively, increasing by $6 \%$ and $12 \%$ over the study period. Patients' QALY gain over the 54 weeks study period was estimated to be 0.029 QALYs. The ANCOVA showed that changes in patients' HRQoL was likely to have been influenced by patients' achievement of blood pressure control, the amount of concomitant medication and patients' last used dosage strength of antihypertensive. Linear regression showed that blood pressure improvement may have been associated with improved HRQOL.

Conclusions: This study showed that OLM/AML/HCTZ reduced blood pressure and significantly increased blood pressure control whilst improving patients' HRQOL. Achieving blood pressure control, amount of concomitant medication and dosage strength of antihypertensive impacted on patients' HRQOL.
\end{abstract}

Keywords: Olmesartan, Adherence, HRQoL, Hypertension, Pill burden

\section{Background}

The risk of cardiovascular disease among hypertensive individuals has been well documented [1], and hypertension for many is an asymptomatic disease. Recent evidence suggests that hypertension may contribute to reduce patients' health-related quality of life (HRQoL) when compared to that of normotensive individuals [2]. Moreover, comorbid diseases in those with arterial hypertension can lead to a worsening of HRQoL when

\footnotetext{
* Correspondence: marco_soro@virgilio.it

${ }^{6}$ Health Economics \& Outcomes Research Department, Daiichi-Sankyo Europe, Munich, Germany

Full list of author information is available at the end of the article
}

compared with hypertensive patients without any comorbidities [2-5]. Some antihypertensive medications have now been shown to worsen the HRQoL of hypertensive patients [6]. However, patients receiving antihypertensive therapy who reach their target blood pressure have an improved HRQoL compared to untreated patients and patients who do not reach their goal [6].

It has been estimated that $15-20 \%$ of hypertensive patients are not adequately controlled on a dual antihypertensive combination and that three or more different antihypertensive drug classes are required to achieve blood pressure control [6-8]. The triple combination antihypertensive therapy olmesartan/amlodipine/hydrochlorothiazide 
(OLM/AML/HCTZ; Sevikar HCT) has been shown to provide significantly greater blood pressure control compared with each of the component dual combinations (i.e. OLM/ AML, OLM/HCTZ and AML/HCTZ) [9].

In a phase III, multicentred, double-blind, parallel-group design study (ClinicalTrials.gov identifier: NCT00923091), 2,690 patients aged $\geq 18$ years with moderate-to-severe hypertension were randomised to receive placebo, OLM/ AML (20 mg/5 mg), OLM/AML (40 mg/5 mg) or OLM/ AML (40 mg/10 mg) for a period of 2 weeks [10]. Patients were then allocated to one of eight groups for a further 8week period by being randomised to continue with the same dose of OLM/AML, or have HCTZ (12.5 mg or $25 \mathrm{mg}$ ) added to their treatment.

The second stage of the study constituted an openlabel extension to assess the long-term efficacy and safety of the triple combination. Of the 2,543 patients who completed the double-blind phase, 2,540 patients initially underwent 8 weeks of single-blind treatment with OLM/AML/HCTZ (20 mg/5 mg/12.5 mg), after which 2,509 were entered into an open-label treatment phase. Of these, 2,439 patients (97.2\%) completed the study [11].

Patients who responded to single-blind treatment with OLM/AML/HCTZ (20 mg/5 mg/12.5 mg) and achieved their target blood pressure (defined as mean seated systolic blood pressure/diastolic pressure (SeSBP/DBP) <140/ $90 \mathrm{mmHg}$, or $<130 / 80 \mathrm{mmHg}$ for those with diabetes or chronic kidney or cardiovascular disease) at week 18 continued with open-label treatment of OLM/AML/HCTZ (20 mg/5 mg/12.5 mg) for 36 weeks [11].

Patients who failed to achieve an adequate response to OLM/AML/HCTZ (20 mg/5 mg/12.5 mg) at week 18 were entered into the first of two consecutive 4-week periods of double-blind treatment which began with rerandomisation (1:2 ratio) to continue with OLM/AML/ HCTZ (20 mg/5 mg/12.5 mg) or up-titration to the $40 \mathrm{mg} / 5 \mathrm{mg} / 12.5 \mathrm{mg}$ dose. At the end of this 4-week period patients whose blood pressure was uncontrolled on OLM/AML/HCTZ (20 mg/5 mg/12.5 mg) were uptitrated to the $40 \mathrm{mg} / 5 \mathrm{mg} / 12.5 \mathrm{mg}$ dose, while those who were controlled continued with their existing treatment. At week 26, all patients who had received the two consecutive 4-week periods of double-blind treatment entered 28 weeks of open-label treatment with OLM/ AML/HCTZ $(40 \mathrm{mg} / 5 \mathrm{mg} / 25 \mathrm{mg})$. Following the start of the open-label treatment phase, patients had their OLM/AML/HCTZ doses titrated with an aim to achieve blood pressure control at the investigator's discretion with either of the following dose combinations: $20 \mathrm{mg} /$ $5 \mathrm{mg} / 12.5 \mathrm{mg}, 40 \mathrm{mg} / 5 \mathrm{mg} / 12.5 \mathrm{mg}, 40 \mathrm{mg} / 5 \mathrm{mg} /$ $25 \mathrm{mg}, 40 \mathrm{mg} / 10 \mathrm{mg} / 12.5 \mathrm{mg}$ or $40 \mathrm{mg} / 10 \mathrm{mg} / 25 \mathrm{mg}$. Up- and down-titration was allowed anytime based on a patient's response to their antihypertensive dose [11].
The 8 week double-blind phase study showed that patients in every triple OLM/AML/HCTZ group had significantly greater mean reductions in diastolic and systolic blood pressure compared with patients on the corresponding OLM/AML therapy dose. During openlabel treatment with OLM/AML/HCTZ, mean SeSBP/ SeDBP levels remained within the ranges 120-140 and 75-85 mmHg, respectively. At the end of the study, significant reductions from baseline were seen in each group for SeSBP (37-43 $\mathrm{mmHg}$ ) and SeDBP (22-27 mmHg), and $78.1 \%$ of patients achieved their blood pressure goal. At baseline, $90.8 \%$ of patients had moderate or severe hypertension, but at the study end $91.9 \%$ had normal/high normal blood pressure. The incidence of adverse events was similar across all treatment groups [11].

The primary aim of this phase III study was to evaluate the efficacy, safety and tolerability of the dual and triple combination therapies, and this has been reported elsewhere [11]. A secondary aim was to measure patients' HRQoL over the study period. In accordance with protocol, of the 2,690 patients who were randomised in the double-blind study [10], 2,679 patients participated in the HRQoL component of the study .

\section{Methods}

\section{Health-related quality of life}

A post-hoc analysis of the data collected during the phase III trial (ClinicalTrials.gov identifier: NCT00923091) enabled an estimation of the HRQoL of 2,690 patients aged $\geq 18$ with moderate-to-severe hypertension who received one of six doses of olmesartan/amlodipine/ hydrochlorothiazide (OLM/AML/HCTZ). Due to the exploratory nature of the analysis, and the fact that the statistical analysis plan was prepared after the clinical report had been completed, the study is subject to several limitations.

The protocol, amendments and informed consent documents were submitted to, and approved by, the Independent Ethics Committee for each centre, prior to study initiation. Written informed consent was received from all patients prior to admission into the study, and investigators ensured that the study was conducted in accordance with the Declaration of Helsinki [10]. Patient reported outcomes were recorded using two healthrelated quality of life instruments: EQ-5D [12] and MINICHAL [13].

The EQ-5D is a generic instrument that measures patients' responses across 5 dimensions of health (mobility, self-care, usual activities, pain/discomfort, and anxiety/ depression). Each dimension has 3 answers to choose from, which can be generally classified as no problems, moderate problems, or significant problems on the specific dimension [12]. A total of 243 possible health states can be defined in this way, each with a unique 5 digit 
code. By applying a formula that attaches weights to each of the levels in each dimension the 5 digit code is converted into a single preference-based health index on a scale from 0 (health state as bad as being dead) to 1 (full health defined by EQ-5D) [12]. The EQ-5D scores were estimated from the preferences of a large general population in the UK [14]. The EQ-5D also includes a visual analog scale which is used to describe a patient's own health state with scores ranging from 0 (worst state) to 100 (best state). When completing the instrument, patients were asked to describe their own health state on the day the questionnaire was answered [12].

The MINICHAL instrument consists of 16 items and measures the impact of hypertension on a patient's HRQoL. The first 10 items are generally grouped to describe a patient's mental state (mood domain) and the last 6 items are designed to evaluate their somatic manifestations (somatic domain), i.e. the potential physical effects of hypertension. Each question has 4 response options: $0=$ No, not at all; $1=$ Yes, somewhat; $2=$ Yes, quite a lot; and $3=$ Yes, a great deal. The score for each dimension is obtained by summing the scores of the items within that dimension and scores range from 0 (best HRQoL) to 30 (worst HRQoL) for the mood domain and from 0 to 18 for the somatic domain [13].

For MINICHAL, patients' completed scores were used to calculate their mood domain, somatic domain, and total scores according to the instrument's specifications and guidelines in order to evaluate changes in patients' HRQoL during the study [13]. For the EQ-5D, patients' scores were converted to utilities according to the instrument's specifications and guidelines [12]. Patients completed these two instruments on four occasions: baseline, week 10, week 26 and week 54 . Changes in HRQoL from baseline to week 54 are reported as changes in the following target variables: EQ-5D index, EQ-5D visual analogue scale, MINICHAL mood domain, MINICHAL somatic domain and MINICHAL total score.

\section{Statistical analyses}

The analyses were conducted in sequential steps.

The first step involved the generation of descriptive statistics for the following quantitative factors:

- Age at baseline ( $\leq 60$ years / elderly $>60$ years);

- Gender;

- Weight based on BMI at baseline (normal $\left[\leq 25 \mathrm{~kg} / \mathrm{m}^{2}\right]$, over-weight [ $>25 \mathrm{~kg} / \mathrm{m}^{2}$ to $\left.\leq 30 \mathrm{~kg} / \mathrm{m}^{2}\right]$ and obese $\left.\left[>30 \mathrm{~kg} / \mathrm{m}^{2}\right]\right) ;$

- Change in systolic blood pressure in quartiles from baseline to Week 54;

- Change in diastolic blood pressure in quartiles from baseline to Week 54;
- Secondary prevention for cardiovascular risk factors (diabetes, cardio-vascular disease, renal impairment);

- Smoking status (Non-Smoker/Ex-Smoker/Current Smoker);

- Country (Eastern EU/Central-Western EU);

- Concomitant cardiovascular risk using concomitant medications as a proxy;

- Mental disorder using concomitant medications as a proxy;

- Number of concomitant medications per patient during the study;

- Baseline hypertension grade (Grade 1 hypertension SBP 140-159 mmHg or DBP 90-99 mmHg; Grade 2 hypertension SBP $160-179 \mathrm{mmHg}$ or DBP 100-109 mmHg; Grade 3 hypertension $\mathrm{SBP} \geq 180 \mathrm{mmHg}$ or DBP $\geq 110 \mathrm{mmHg}$ );

- Blood pressure goal control at week 54 (SBP/ DBP $\leq 130 / 80 \mathrm{mmHg}$ for diabetic patients, renally impaired patients, or patients with cardiovascular disease; SBP $\leq 140 / 90 \mathrm{mmHg}$ for all other patients).

The second step involved performing analysis of covariance (ANCOVA) for each target variable and factor, to assess if these factors possibly influenced HRQoL changes during the study. Factors with p-value of $>0.2$ were omitted in the third step. Any factors with a pvalue for effect of 0.2 or lower were used in the third step for the respective target covariate. The third step involved stepwise ANCOVA that included treatment and all pre-selected factors for each target variable based on second step findings until all remaining factors had $\mathrm{p}$ values $\leq 0.05$.

The outputs of the analyses were a descriptive summary of the results. P-values between 0.05 and 0.01 were considered a trend and p-values of 0.01 or lower were considered to be indicative. Since multiple tests were performed without any adjustment, the p-values even if statistically significant, should only be considered to be indicative of trends or possible hypotheses.

Since blood pressure changes from baseline to week 54 were initially not included in the ANCOVA models, a separate linear regression model was calculated to assess the relationship between changes in blood pressure and HRQoL scores from baseline.

\section{Results}

Of the 2,690 patients who were randomised into the double-blind study [10], 2,679 patients participated in the HRQoL component of the study. The demographic and baseline characteristics of the 2,679 patients who completed the HRQoL instruments at baseline are shown in Table 1. The mean age of patients was 56.2 years; mean weight was $88.5 \mathrm{~kg}$; $53.7 \%$ were female [11]; $14.6 \%$ were diabetic; and $28.70 \%$ had cardiovascular 
Table 1 Patients' characteristics

\begin{tabular}{ll}
\hline & $\begin{array}{l}\text { Number of patients (percentage } \\
\text { of sample in parentheses) }\end{array}$ \\
\hline Age & $1743(65.1 \%)$ \\
$<=60$ years & $936(34.9 \%)$ \\
Gender & \\
Female & $1439(53.7 \%)$ \\
Male & $1240(46.3 \%)$ \\
Body mass index & \\
$<=25 \mathrm{~kg} / \mathrm{m} 2$ & $309(11.5 \%)$ \\
$>25$ to $<=30 \mathrm{~kg} / \mathrm{m} 2$ & $1003(37.4 \%)$ \\
$=>30 \mathrm{~kg} / \mathrm{m} 2$ & $1367(51.0 \%)$ \\
Smoking status & \\
Current smoker & $464(17.3 \%)$ \\
Ex-smoker & $301(11.2 \%)$ \\
Non-smoker & $1914(71.4 \%)$ \\
Country & \\
Eastern European \\
countries \\
Western-central \\
European countries
\end{tabular}

disease (Table 2). The mean baseline SeSBP/SeDBP was $168.4 / 103.8 \mathrm{mmHg}$ and $89.7 \%$ of patients had SBP between 140 and $179 \mathrm{mmHg}$ or DBP between 90 and $109 \mathrm{mmHg}$ (Grade 1 or 2 hypertension) whilst 10.3\% had SBP of 180 or DBP of $110 \mathrm{mmHg}$ (Grade 3 hypertension) and the mean duration of disease (hypertension) was 8.3 years.

The efficacy and safety of the alternative antihypertensive combinations have been described elsewhere $[10,11]$. However, it is noteworthy that differences were observed in the baseline characteristics of patients who ended the study in different treatment groups. For example, the low dose OLM/AML/HCTZ 20/5/12.5 mg group contained the lowest proportion of males, current smokers, diabetics and patients with cardiovascular disease. In contrast, the higher dose OLM/AML/HCTZ 40/ $10 / 12.5$ and 40/10/25 mg groups contained more males and higher proportions of diabetics and patients with cardiovascular disease.

At baseline patients' MINICHAL mood domain and somatic domain scores were 5.5 and 2.6 respectively. Over the 54 weeks study period patients' HRQoL improved as both MINICHAL scores decreased by 31-33\% (Figure 1). Patients' baseline EQ-5D index and VAS score was 0.9 and 73.4 respectively, (Figure 2) increasing by $6 \%$ and $12 \%$ respectively over the study period (Table 3). The mean difference in the EQ-5D index score from baseline to week 54 was assessed with a univariate
Table 2 Patients' clinical profile

\begin{tabular}{ll}
\hline & $\begin{array}{l}\text { Number of patients (percentage } \\
\text { of sample in parentheses) }\end{array}$ \\
\hline Baseline hypertension & $27(1.0 \%)$ \\
Grade 1 & $2375(88.7 \%)$ \\
Grade 2 & $277(10.3 \%)$ \\
Grade 3 & \\
Diabetes & $2287(85.4 \%)$ \\
No & $392(14.6 \%)$ \\
Yes & \\
Cardiovascular \\
disease
\end{tabular}

procedure to calculate the change in quality-adjusted life year (QALY). Patients' QALY gain over the 54 weeks study period was estimated to be 0.029 QALYs.

The majority of patients reported no problems in the different EQ-5D dimensions, although more patients reported experiencing pain/discomfort and anxiety/ depression than problems with the other dimensions (Figure 3).

Results from Step 2 of the ANCOVA showed that renal impairment and smoking status were unlikely to have influenced the HRQoL changes observed during the study, since their $\mathrm{p}$ value was $>0.2$. Hence, they were omitted from the ANCOVA model in the third step. 


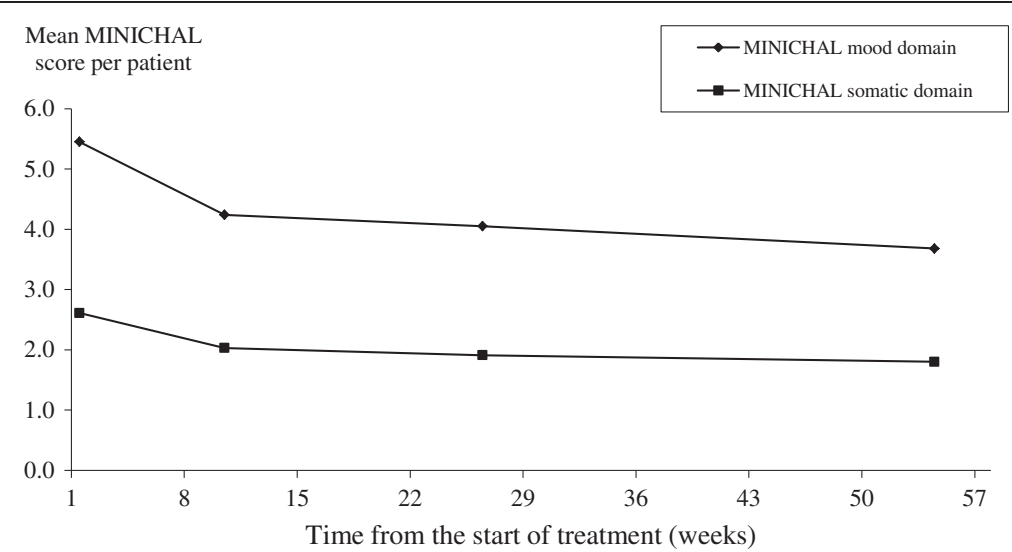

Figure 1 Mean MINICHAL scores per patient at different times from the start of antihypertensive treatment. Not all patients completed all HRQOL instruments at each time point.

Blood pressure changes in quartiles were also excluded from the third step as that was pre-determined by the analysis protocol. The factors/endpoints with a $\mathrm{p}$ value of $<0.05$ after performing Step 3 of the ANCOVA are summarised in Tables 4 and 5. Whilst these p values are explorative only, they are indicative of those baseline factors that influenced the patients' HRQoL status at the end of the study period. Nevertheless, there were very slight differences in the change in patients' HRQoL over the study period when stratified by the different baseline factors. For example, the change in HRQoL was similar irrespective of patients' age, gender, BMI, etc. The one exception to this was in patients' baseline hypertension grade. The change in HRQoL for patients with Grade 2 and 3 hypertension was similar, but different to that of patients with Grade 1 when assessed using the EQ-5D VAS. However, patients' baseline hypertension grade was found not to have influenced HRQoL when assessed using the MINICHAL. Similarly, neither the presence of cardiovascular disease nor patients' use of concomitant medication for cardiovascular disease or mental disorder appeared to influence their HRQoL when assessed by MINICHAL. Conversely, the amount of concomitant medication ("pill burden"), blood pressure target achievement and patients' antihypertensive treatment in period 6 influenced HRQoL when assessed by the EQ$5 \mathrm{D}$ but not when assessed by the MINICHAL. Notwithstanding this, patients' baseline HRQoL score derived from each scale influenced their HRQoL score on that same scale at week 54 .

The factors included in the EQ-5D VAS step 3 model are not exhaustive and do not account for all of the observed difference in VAS score. The $R^{2}$ for the ANCOVA model was 0.188. The different factors incorporated in the separate EQ-5D index, MINICHAL mood and MINICHAL somatic step 3 models are likely to be more influential over the changes in patients' HRQoL and the $R^{2}$ was $0.408,0.431$ and 0.448 respectively. Notwithstanding this, changes in the patients' HRQoL was possibly influenced by other factors which were not measured in this study. Additionally, a separate linear regression analysis showed that blood pressure improvement may have been associated with improved HRQoL.

\section{Discussion}

This study assessed the HRQoL of hypertensive patients who received one of six doses of OLM/AML/HCTZ to reduce their high blood pressure using both the EQ-5D and MINICHAL instruments over 54 weeks including the open label phase. At the start of the study, $90.8 \%$ of patients had Grade 2 or 3 hypertension, but at the study end $91.9 \%$ had achieved the SeSBP $<140 \mathrm{mmHg}$ threshold [11]. Furthermore, the incidence of adverse events was similar across the treatment groups [11]. Patients' hypertension grade at baseline did not appear to affect their HRQoL, except the EQ-5D VAS score of those with Grade 3 disease was lower than that of the other patients. This may reflect the admission criteria of the clinical trial $[10,11]$ since patients' baseline health status was relatively high. In contrast, others have reported that patients with Grade 1 disease have better MINICHAL scores than those with Grade 2/3 [15,16].

Previous studies using the MINICHAL have shown that patients treated with antihypertensives who experienced reduced blood pressure experienced a greater decrease in MINICHAL scores than those patients who reported no overall change in blood pressure. In this study, patients' HRQoL improved since their MINICHAL scores decreased by $31-33 \%$ over the 54 weeks study period. However, what constitutes clinically relevant changes in MINICHAL scores is currently undecided. The mean mood and somatic MINICHAL scores among a normotensive population in Brazil have been estimated at 3.2 and 0.8 respectively [17]. The corresponding values 


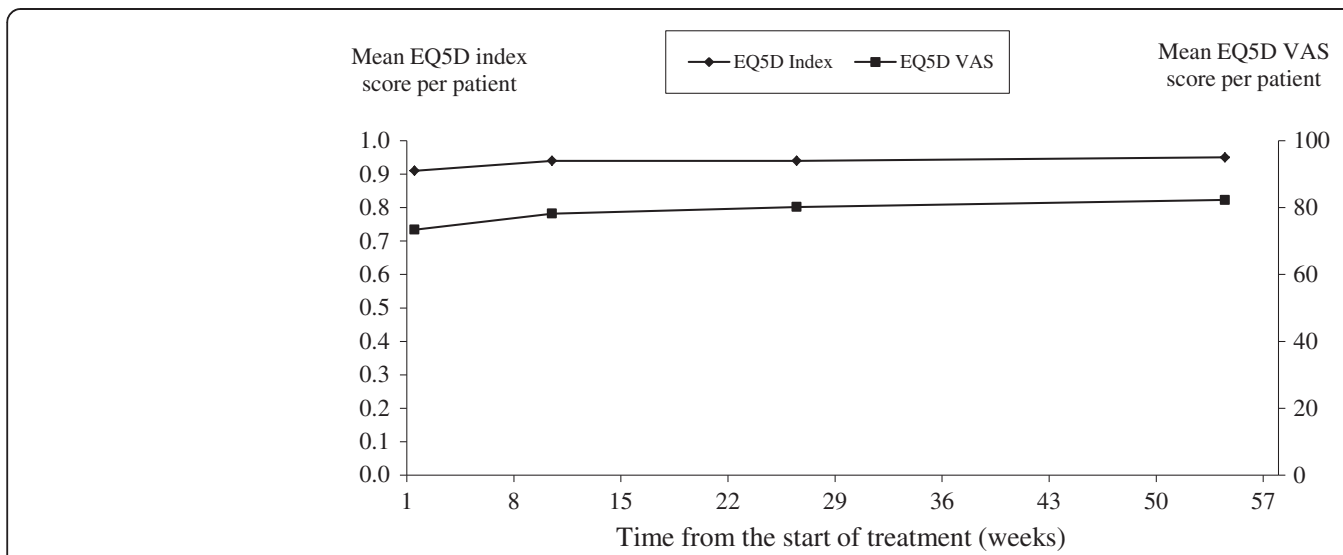

Figure 2 Mean EQ-5D scores per patient at different times from the start of antihypertensive treatment. Not all patients completed all HRQOL instruments at each time point.

among the hypertensive population in the Brazilian study was 5.3 and 1.9 respectively [17]. Other studies that have used the MINICHAL reported mean mood scores of 6.6 [18] and 5.6 [15] and mean somatic scores of 5.0 [18] and 2.1 [15]. In the present study the mean mood and somatic baseline scores of 5.5 and 2.6 respectively were concordant with those from the other studies $[15,17,18]$. Notwithstanding the variance between studies, use of the MINICHAL detected that the patients in the present study had a poorer HRQoL than would be expected from normotensive patients, which is consistent with the finding of others [19]. Moreover, the somatic domain appeared to be more sensitive than the mood domain in the detecting changes in patients' HRQoL in this study.

The ANCOVA model showed that multiple factors influenced HRQoL changes. However, individual categorical variables (as identified in step 3) would seem to have only accounted for up to $40 \%$ of the variation in patients' HRQoL (measured using the MINICHAL), since $\mathrm{R}^{2}$ was of the order of 0.4. This value indicates that other variables (such as socioeconomic factors and stress which were not measured in the clinical trials) have not been included in the ANCOVA model and thus it was not possible to control for their effect. Nevertheless, achievement of blood pressure target was found to be an influencer for HRQoL. Both these variables have been shown to be independent predictors of $\mathrm{HRQoL}$ among hypertensive patients [20,21]. Also, step 3 of the MINICHAL model suggests that the amount of co-medication ("pill burden") affects patients' HRQoL. Moreover, a high pill burden among hypertensive patients has been shown to adversely affect adherence [22,23]. Therefore, the findings from this study support the notion that physicians should prescribe in a manner to reduce their patients' pill burden. Additionally, the HRQoL of patients treated with an anti-hypertensive in an observational study was found to significantly influence adherence to treatment [24]. Furthermore, patients with high adherence to their treatment schedule have a much higher chance of attaining their target blood pressure than patients with low adherence [25]. Consequently, adherence plays an important role in reducing the risk of developing cardiovascular events [26]. Moreover, achieving blood pressure control influenced patients' HRQoL, as demonstrated by the step 3 EQ-5D index and MINICHAL somatic domain models, despite patients' high baseline health status.

The mean EQ-5D index and VAS baseline scores for the whole study cohort was 0.9 and 73.4 respectively.

Table 3 Patients' mean (standard deviation) blood pressure and health-related quality of life status*

\begin{tabular}{lllll}
\hline & Baseline & Week 54 & $\begin{array}{l}\text { Difference between week } \\
\mathbf{5 4} \text { and baseline }\end{array}$ & $\begin{array}{c}\text { p value derived } \\
\text { using a t-test }\end{array}$ \\
\hline Systolic blood pressure & $168.4(6.6)$ & $127.1(9.0)$ & $-41.3(10.0)$ & $<0.0001$ \\
Diastolic blood pressure & $103.8(2.9)$ & $78.0(6.6)$ & $-25.9(6.9)$ & $<0.0001$ \\
EQ-5D index & $0.90(0.13)$ & $0.95(0.11)$ & $0.05(0.12)$ & $<0.0001$ \\
EQ-5D VAS & $73.4(15.0)$ & $82.3(12.5)$ & $8.9(14.4)$ & $-1.80(4.24)$ \\
MINICHAL mood domain & $5.45(4.42)$ & $3.68(3.74)$ & $-0.80(2.46)$ & $<0.0001$ \\
MINICHAL somatic domain & $2.62(2.66)$ & $1.80(2.16)$ & $-2.60(5.68)$ & $<0.0001$ \\
MINICHAL total score & $8.00(6.22)$ & $5.40(5.12)$ & & $<0.0001$ \\
\hline
\end{tabular}

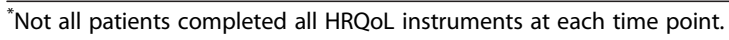




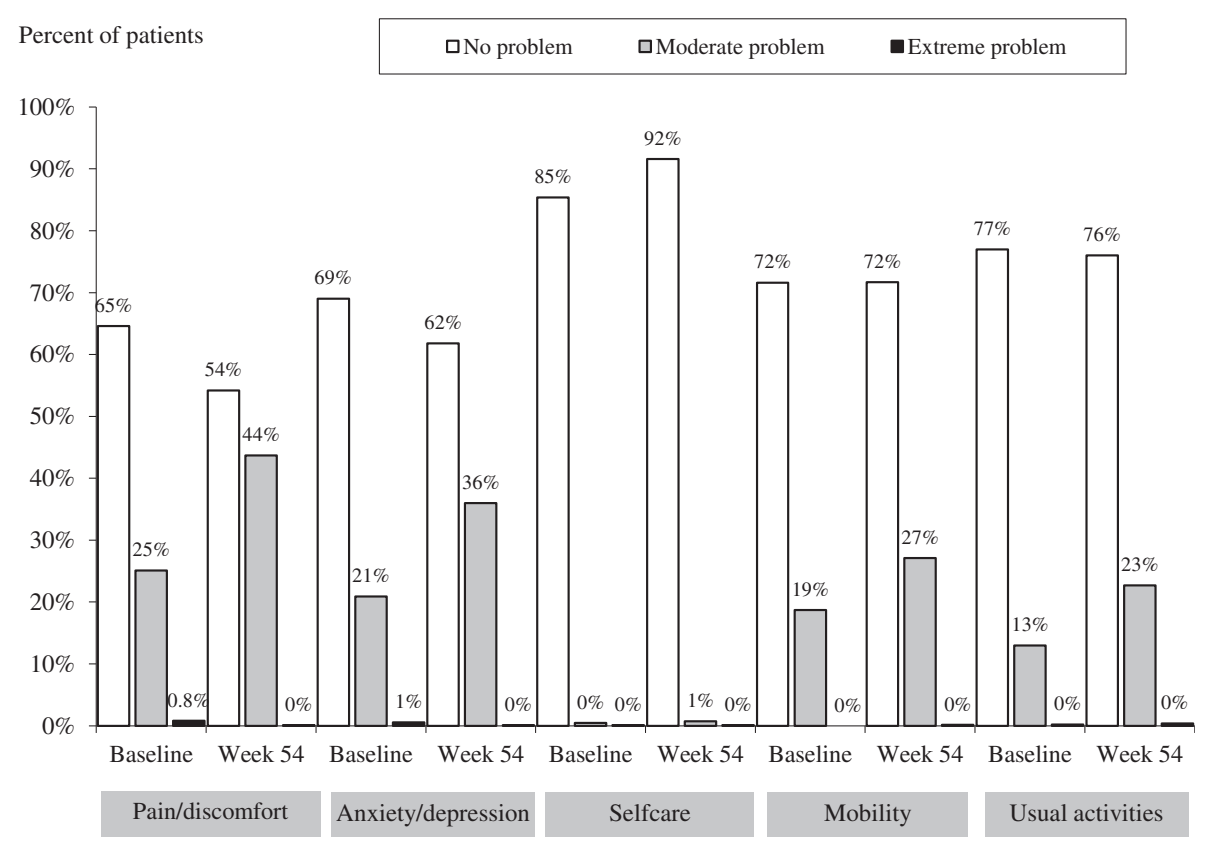

Figure 3 Percentages of patients reporting problems across each EQ-5D index dimension at baseline and after 54 weeks of treatment. Data was missing from $10 \%$ of patients at baseline and $1 \%$ of patients at week 54 .

These baseline scores are high, probably due to the fact that patients with major concomitant cardiovascular risk factors and co-morbidities were excluded by the clinical trial protocol. Nonetheless, these scores increased by $6 \%$ and $12 \%$ respectively over the study period. In comparison, the international population norm for the EQ-5D index across the 18 to $75+$ age group has been reported to range from 0.74 to 0.95 and that of the EQ-5D VAS has been shown to range from 71.1 to 83.7 [27]. These comparisons suggest that the EQ-5D index score of this patient population is higher relative to population norms than the EQ-5D VAS, which may suggest that the EQ$5 \mathrm{D}$ index is not a sufficiently sensitive instrument with which to measure HRQoL among hypertensive patients with no major additional cardiovascular risk factors.

Notwithstanding this, the analysis showed that age, gender, BMI, whether patients were taking cconcomitant medication for cardiovascular disease and/or mental disorder and whether they achieved hypertension control by week 54 all influenced the EQ-5D index score. Additionally, gender, co-morbid diabetes, co-morbid cardiovascular disease, country of origin, whether patients were taking concomitant medication for mental disorder and their hypertension grade at baseline influenced their EQ-5D VAS score. The effect of concomitant cardiovascular diseases on HRQoL has been much debated in the published literature. For example, the epidemiological study ESTHER showed that in a large cohort of hypertensive patients being managed by general practitioners, additional cardiovascular risk factors did not seem to be related to a substantial worsening of their HRQoL measured using the SF-12. Nonetheless, the physical component score (PCS) reported in the ESTHER study was found to be significantly decreased by cardiovascular risk factors such as BMI, macroalbuminuria, diabetes and circulatory disorders [28]. In our phase III study study on OLM/AML/HCTZ the analysis of patients' comorbidities suggested that the study population comprised a relatively healthy cohort with relatively few experiencing comorbid illness, reflecting the exclusion criteria of the trial. The possibility of a "ceiling effect" in EQ-5D index because of the high baseline values (37.3\% of patients had an EQ-5D value of 1.00) leading to artifactual parameter estimates in the ANCOVA cannot be excluded. It was not considered appropriate to reduce the potential impact of a ceiling effect as available econometric methods [29] have been applied to simple ordinary least squares regression models, but not to ANCOVA models that incorporate covariates. Notwithstanding the limitations of using the EQ-5D instrument to detect HRQoL changes among hypertensive patients, it did detect important differences in those who had controlled their hypotension.

The Step 3 analyses identified multiple factors that were likely to have influenced HRQoL simultaneously. Blood pressure control was the only blood pressurerelated factor modelled in Step 3. However, linear regression showed that blood pressure improvement may have been associated with improved HRQoL.

This post-hoc analysis is subject to several other limitations. The statistical analysis protocol for the post-hoc 
Table 4 Results of Step 3 from the ANCOVA investigating the relationship between sociodemographic factors and morbidity on patients' health-related quality of life measured using the EQ-5D

\begin{tabular}{lll}
\hline Endpoint/Factors & EQ-5D Index & \\
\cline { 2 - 3 } & Baseline & $\begin{array}{l}\text { Difference } \\
\text { week 54 and }\end{array}$ \\
\hline $\begin{array}{l}\text { Age class } \\
\quad=60 \text { years } \\
>60 \text { years }\end{array}$ & $0.92(0.12)$ & $0.00(0.12)$ \\
Gender & $0.89(0.14)$ & $0.00(0.13)$ \\
Male & & \\
Female & $0.94(0.11)$ & $0.00(0.10)$ \\
BMl & $0.89(0.15)$ & $0.00(0.14)$ \\
$<=25 \mathrm{~kg} / \mathrm{m} 2$ & & \\
$>25$ to $<=30 \mathrm{~kg} / \mathrm{m} 2$ & $0.94(0.11)$ & $0.00(0.12)$ \\
$=>30 \mathrm{~kg} / \mathrm{m} 2$ & $0.92(0.13)$ & $0.00(0.12)$
\end{tabular}

Diabetes

Yes

No

Cardiovascular disease

Yes

No

Country (region)

Western-central countries Eastern countries

Concomitant medication for cardiovascular disease

Yes

No

Concomitant medication for mental disorder

$$
\text { Yes }
$$

No

Hypertension grade at

baseline

Grade 1

Grade 2
EQ-5D VAS

Baseline

Difference between

week 54 and baseline

p value

$<0.0001$

0.0005

0.0002

75.6 (14.2)

-8.50 (13.59)

71.4 (15.4)

$-9.30(15.08)$

0.0426

$\begin{array}{ll}69.2(15.4) & -8.60(15.91) \\ 74.1(14.8) & -8.90(14.14) \\ 70.4(14.5) & -10.90(13.83) \\ 74.5(15.5) & -8.10(14.55)\end{array}$

$<0.0001$

0.0085

$76.0(15.5) \quad-7.80(15.50)$

$72.0(14.5) \quad-9.40(13.81)$

0.0065

$\begin{array}{ll}0.91(0.13) & 0.00(0.12) \\ 0.92(0.12) & 0.00(0.13)\end{array}$

$0.00(0.13)$

0.021

0.0263

$0.88(0.17) \quad 0.00(0.19)$

$0.92(0.13) \quad 0.00(0.12)$

$\begin{array}{ll}68.7(17.8) & -10.40(18.05) \\ 73.6(14.7) & -8.80(14.22) \\ & \\ 80.6(11.8) & -1.10(11.09) \\ 73.4(15.0) & -8.80(14.25)\end{array}$


Table 4 Results of Step 3 from the ANCOVA investigating the relationship between sociodemographic factors and morbidity on patients' health-related quality of life measured using the EQ-5D (Continued)

\begin{tabular}{|c|c|c|c|c|c|c|}
\hline Grade 3 & & & & $72.4(15.2)$ & $-10.40(15.67)$ & \\
\hline $\begin{array}{l}\text { Achieved hypertension } \\
\text { control by week } 54\end{array}$ & & & 0.0019 & & & \\
\hline Yes & $0.92(0.13)$ & $0.00(0.12)$ & & & & \\
\hline No & $0.89(0.15)$ & $0.00(0.14)$ & & & & \\
\hline $\begin{array}{l}\text { Baseline value of respective } \\
\text { endpoint }\end{array}$ & & & $<0.0001$ & & & $<0.0001$ \\
\hline $\begin{array}{l}\text { SeSBP changes from baseline } \\
\text { to week } 54\end{array}$ & & & 0.0010 & & & 0.0160 \\
\hline $\begin{array}{l}\text { SeDPB changes from baseline } \\
\text { to week } 54\end{array}$ & & & 0.0039 & & & 0.0120 \\
\hline
\end{tabular}


Table 5 Results of Step 3 from the ANCOVA investigating the relationship between sociodemographic factors and morbidity on patients' health-related quality of life measured using the MINICHAL*

\begin{tabular}{|c|c|c|c|c|c|c|c|c|c|}
\hline \multirow[t]{2}{*}{ Endpoint/Factors } & \multicolumn{3}{|c|}{ MINICHAL mood domain } & \multicolumn{3}{|c|}{ MINICHAL somatic domain } & \multicolumn{3}{|c|}{ MINICHAL total score } \\
\hline & Baseline & $\begin{array}{l}\text { Difference between } \\
\text { week } 54 \text { and baseline }\end{array}$ & $\mathrm{p}$ value & Baseline & $\begin{array}{l}\text { Difference between } \\
\text { week } 54 \text { and baseline }\end{array}$ & $\mathrm{p}$ value & Baseline & $\begin{array}{l}\text { Difference between } \\
\text { week } 54 \text { and baseline }\end{array}$ & $p$ value \\
\hline Age class & & & $<0.0001$ & & & 0.0058 & & & $<0.0001$ \\
\hline$<=60$ years & $5.34(4.38)$ & $-2.00(4.21)$ & & $2.51(2.68)$ & $-0.80(2.46)$ & & $7.80(6.24)$ & $-2.80(5.67)$ & \\
\hline$>60$ years & $5.63(4.48)$ & $-1.40(4.27)$ & & $2.81(2.61)$ & $-0.70(2.45)$ & & $8.38(6.16)$ & $-2.00(5.66)$ & \\
\hline Gender & & & 0.0151 & & & 0.0004 & & & 0.0045 \\
\hline Male & $4.74(4.10)$ & $-1.60(4.00)$ & & $2.18(2.34)$ & $-0.70(2.22)$ & & $6.88(5.66)$ & $-2.30(5.38)$ & \\
\hline Female & $6.06(4.59)$ & $-1.90(4.43)$ & & $2.99(2.86)$ & $-0.90(2.64)$ & & $8.99(6.51)$ & $-2.80(5.92)$ & \\
\hline BMI & & & & & & 0.0063 & & & \\
\hline$<=25 \mathrm{~kg} / \mathrm{m} 2$ & & & & $2.32(2.50)$ & $-0.80(2.03)$ & & & & \\
\hline$>25$ to $<=30 \mathrm{~kg} / \mathrm{m} 2$ & & & & $2.33(2.53)$ & $-0.80(2.40)$ & & & & \\
\hline$=>30 \mathrm{~kg} / \mathrm{m} 2$ & & & & $2.89(2.76)$ & $-0.80(2.58)$ & & & & \\
\hline Diabetes & & & 0.0326 & & & & & & \\
\hline Yes & $5.80(4.70)$ & $-1.40(4.52)$ & & & & & & & \\
\hline No & $5.38(4.37)$ & $-1.90(4.19)$ & & & & & & & \\
\hline Country (region) & & & $<0.0001$ & & & & & & $<0.0001$ \\
\hline $\begin{array}{l}\text { Western-central } \\
\text { countries }\end{array}$ & $4.56(4.66)$ & $-1.80(4.42)$ & & & & & $6.70(6.45)$ & $-2.30(5.97)$ & \\
\hline Eastern countries & $5.90(4.22)$ & $-1.80(4.14)$ & & & & & $8.68(5.98)$ & $-2.70(5.52)$ & \\
\hline $\begin{array}{l}\text { Amount of concomitant } \\
\text { medication }\end{array}$ & & & 0.0387 & & & $<0.0001$ & & & 0.0002 \\
\hline 0 & $5.79(4.27)$ & $-2.20(4.30)$ & & $2.43(2.42)$ & $-1.00(2.05)$ & & $8.21(5.77)$ & $-3.20(5.24)$ & \\
\hline $1-2$ & $5.44(4.32)$ & $-1.90(3.92)$ & & $2.32(2.55)$ & $-0.80(2.38)$ & & $7.69(6.00)$ & $-2.70(5.25)$ & \\
\hline $3-4$ & $5.15(4.22)$ & $-1.80(4.02)$ & & $2.73(2.78)$ & $-0.80(2.53)$ & & $6.00(5.06)$ & $-2.50(5.70)$ & \\
\hline $5-6$ & $5.38(4.37)$ & $-1.30(4.40)$ & & $2.91(2.78)$ & $-0.70(2.55)$ & & $8.28(6.22)$ & $-2.00(5.97)$ & \\
\hline$=>7$ & $5.61(5.08)$ & $-1.50(5.01)$ & & $3.01(2.75)$ & $-0.60(2.85)$ & & $8.57(7.00)$ & $-2.00(6.77)$ & \\
\hline $\begin{array}{l}\text { Achieved hypertension } \\
\text { control by week } 54\end{array}$ & & & & & & 0.002 & & & 0.011 \\
\hline Yes & & & & $2.44(2.59)$ & $-0.80(2.37)$ & & $7.75(6.13)$ & $-2.70(5.62)$ & \\
\hline No & & & & $3.13(2.85)$ & $-0.70(2.77)$ & & $8.67(6.33)$ & $-2.20(5.90)$ & \\
\hline Treatment in Period 6 & & & 0.0158 & & & 0.0012 & & & 0.024 \\
\hline OM20/AML5/HCTZ12.5 & $5.19(4.24)$ & $-1.90(4.23)$ & & $2.39(2.59)$ & $-0.80(2.32)$ & & $7.48(5.99)$ & $-2.60(5.59)$ & \\
\hline OM40/AML5/HCTZ12.5 & $5.94(5.05)$ & $-1.40(4.80)$ & & $3.02(3.04)$ & $-1.00(3.02)$ & & $8.93(7.09)$ & $-2.40(6.81)$ & \\
\hline
\end{tabular}


Table 5 Results of Step 3 from the ANCOVA investigating the relationship between sociodemographic factors and morbidity on patients' health-related quality of life measured using the MINICHAL* (Continued)

\begin{tabular}{|c|c|c|c|c|c|c|c|c|c|}
\hline OM40/AML5/HCTZ25 & $5.94(4.58)$ & $-2.00(4.12)$ & & $2.67(2.58)$ & $-0.90(2.49)$ & & $8.59(6.22)$ & $-2.90(5.50)$ & \\
\hline OM40/AML10/HCTZ12.5 & $5.69(4.39)$ & $-1.70(3.90)$ & & $3.19(2.95)$ & $-0.50(2.77)$ & & $8.95(6.46)$ & $-2.30(5.88)$ & \\
\hline OM40/AML10/HCTZ25 & $4.83(3.89)$ & $-1.20(3.88)$ & & $2.86(2.43)$ & $-0.50(2.25)$ & & $7.67(5.41)$ & $-2.00(4.73)$ & \\
\hline $\begin{array}{l}\text { Baseline value of } \\
\text { respective endpoint }\end{array}$ & & & $<0.0001$ & & & $<0.0001$ & & & $<0.0001$ \\
\hline $\begin{array}{l}\text { SeSBP changes from } \\
\text { baseline to week } 54\end{array}$ & & & 0.0089 & & & 0.0002 & & & 0.0006 \\
\hline $\begin{array}{l}\text { SeDPB changes from } \\
\text { baseline } \\
\text { to week } 54\end{array}$ & & & 0.1089 & & & 0.0080 & & & 0.0273 \\
\hline
\end{tabular}


HRQoL analysis was prepared after the clinical report had been completed. Multiple statistical tests were performed without any adjustment. Hence, the p-values can only be viewed as being exploratory and indicative of trends or possible hypotheses. Furthermore, the large sample size may have resulted in small, statistically significant $\mathrm{p}$ values for changes that may have marginal clinical significance. Factors that may have influenced patients' HRQoL, such as educational level, marital status, job status, income and residency, were not collected during the phase III trial. Exclusion of these factors should be considered as an additional limitation. Moreover, the large sample size of this non-comparative study has only generated small effects. Hence, the reproducibility of the HRQoL impact of OLM/AML/HCTZ seen in this study needs to be studied further in a controlled study design.

In conclusion, this present study showed that OLM/ AML/HCTZ reduced blood pressure and significantly increased blood pressure control whilst improving patients' HRQoL. Achieving blood pressure control, amount of concomitant medication and dosage strength of antihypertensive all impacted on patients' HRQoL. Hence, when prescribing antihypertensive agents physicians should consider the impact of all these factors on patients' HRQoL and on their adherence to treatment.

\section{Competing interests}

This study was supported with an unrestricted research grant from Daiichi Sankyo Europe GmbH, Munich, Germany.

P Marques da Silva and J Brazier received honoraria for their participation in this study. U Haag's and JF Guest's organisation received honoraria for their participation in this study. M Soro is a current employee of Daiichi Sankyo Europe $\mathrm{GmbH}$, Munich, Germany.

The authors have no other conflict of interests that are directly relevant to the content of this paper, which remains their sole responsibility.

\section{Authors' contributions}

PMS interpreted the clinical data. UH carried out the statistical analysis. JG interpreted the clinical and statistical data and wrote the manuscript. JB interpreted the HRQoL data. MS designed the post-hoc analysis and reviewed the manuscript. All authors read and approved the final manuscript.

\section{Author details}

'Arterial Investigation Unit, Medicine 4, Santa Marta's Hospital, CHLC, Lisbon, Portugal. ${ }^{2} \mathrm{HaaPACS} \mathrm{GmbH}$, Schriesheim, Germany. ${ }^{3}$ Catalyst Health Economics Consultants, Northwood, UK. ${ }^{4}$ Faculty of Life Sciences and Medicine, King's College, London, UK. ${ }^{5}$ Health Economics and Decision Science (HEDS), School of Health and Related Research (ScHARR), University of Sheffield, Sheffield, UK. ${ }^{6}$ Health Economics \& Outcomes Research Department, Daiichi-Sankyo Europe, Munich, Germany.

Received: 1 September 2014 Accepted: 27 January 2015

Published online: 21 February 2015

\section{References}

1. Jansen $H$, Samani NJ, Schunkert H. Mendelian randomization studies in coronary artery disease. Eur Heart J. 2014. ([Epub ahead of print]). Available from: http://www.ncbi.n/m.nih.gov/pubmed/24917639.

2. Trevisol DJ, Moreira LB, Kerkhoff A, Fuchs SCFF. Health-related quality of life and hypertension: a systematic review and meta-analysis of observational studies. J Hypertens. 2011;29(2):179-88.
3. Zygmuntowicz M, Owczarek A, Elibol A, Chudek J. Comorbidities and the quality of life in hypertensive patients. Pol Arch Med Wewnętrznej. 2012;122(7-8):333-40.

4. Wang $R$, Zhao $Y$, He $X$, Ma $X$, Yan $X$, Sun $Y$, et al. Impact of hypertension on health-related quality of life in a population-based study in Shanghai. China Pub Health. 2009;123:534-9.

5. Aydemir O, Ozdemir C, Koroglu E. The impact of co-morbid conditions on the SF-36: A primary-care-based study among hypertensives. Arch Med Res. 2005;36:136-41.

6. Erickson SR, Williams BC, Gruppen LD. Perceived symptoms and health-related quality of life reported by uncomplicated hypertensive patients compared to normal controls. J Hum Hypertens. 2001;15:539-48.

7. Mancia G, De Backer G, Dominiczak A, Cifkova R, Fagard R, Germano G, et al. Guidelines for the management of arterial hypertension: the task force for the management of arterial hypertension of the european society of hypertension (ESH) and of the european society of cardiology (ESC). J Hypertens. 2007;25(6):1105-87.

8. Mancia G, Laurent S, Agabiti-Rosei E, Ambrosioni E, Burnier M, Caulfield MJ, et al. Reappraisal of european guidelines on hypertension management: a european society of hypertension task force document. J Hypertens. 2009;27(11):2121-58.

9. Oparil S, Melino M, Lee J, Fernandez V, Heyrman R. Triple therapy with olmesartan medoxomil, amlodipine besylate, and hydrochlorothiazide in adult patients with hypertension: the TRINITY multicenter, randomized, double-blind, 12-week, parallel-group study. Clin Ther. 2010;32:1252-69.

10. Volpe $M$, Christian Rump L, Ammentorp B, Laeis P. Efficacy and safety of triple antihypertensive therapy with the olmesartan/amlodipine/ hydrochlorothiazide combination. Clin Drug Investig. 2012;32(10):1.

11. Volpe M, de la Sierra A, Ammentorp B, Laeis P. Open-label study assessing the long-term efficacy and safety of triple olmesartan/amlodipine/hydrochlorothiazide combination therapy for hypertension. Adv Ther. 2014;31(5):561-74.

12. EuroQol. EQ-5D. Available from: http://www.euroqol.org/.

13. Badia X, Roca-Cusachs A, Dalfo A, Gascon G, Abellan J, Lahoz R, et al. Validation of the short form of the Spanish Hypertension Quality of Life Questionnaire (MINICHAL). Clin Ther. 2002;24(12):2137-54.

14. Dolan P. Modeling valuations for EuroQol health states. Med Care. 1997;35:1095-108.

15. Roca-cusachs À, Badia X, Dalfó A, Gascón G, De Bienestar Î. Relación entre variables clínicas y terapéuticas y calidad de vida relacionada con la salud en pacientes con hipertensión arterial. Estudio MINICHAL. Med Clin. 2003;121(1):12-7.

16. Schulz RB, Rossignoli P, Correr CJ, Fernández-Llimós FTP. Validation of the short form of the spanish hypertension quality of life questionnaire ( MINICHAL) for Portuguese ( Brazil). Arq Bras Cardiol. 2008;90(2):127-31.

17. Carvalho MA, Silva IB, Ramos SB, Coelho LF, Goncalves ID, Figueiredo Neto $J A$, et al. Quality of life of hypertensive patients and comparison of two instruments of HRQOL measure. Arq Bras Cardiol. 2012;98(5):442-50.

18. Roca-Cusachs A, Dalfó A, Badia X, Arístegui I, Roset M. Relation between clinical and therapeutic variables and quality of life in hypertension. J Hypertens. 2001;19(10):1913-9.

19. Youssef RM, Moubarak II, Kamel MI. Factors affecting the quality of life of hypertensive patients. East Mediterr Heal J. 2005;11(1-2):109-18.

20. Santos L, Chaves C, Andrade A, Duarte J. The influence of stress on the quality of life of hypertensive patients. Procedia - Soc Behav Sci Elsevier B V. 2014;113:16-25.

21. Panjabi S, Lacey M, Bancroft T, Cao F. Treatment adherence, clinical outcomes, and economics of triple-drug therapy in hypertensive patients. J Am Soc Hypertens. 2013;7(1):46-60.

22. Antihypertensive Pill Burden \& Patient Adherence. Available from: http://www physiciansweekly.com/ash-2014-antihypertensive-pill-burden-adherence/.

23. Zyoud SH, Al-Jabi SW, Sweileh WM, Wildali AH, Saleem HM, Aysa HA, et al. Health-related quality of life associated with treatment adherence in patients with hypertension: a cross-sectional study. Int J Cardiol. 2013;168(3):2981-3.

24. DiMatteo MR, Giordani PJ, Lepper HSCT. Patient adherence and medical treatment outcomes: a meta-analysis. Med Care. 2002;40(9):794-811.

25. Ho PM, Magid DJ, Shetterly SM, Olson KL, Maddox TM, Peterson PN, et al. Medication nonadherence is associated with a broad range of adverse outcomes in patients with coronary artery disease. Am Heart J. 2008;155 (4):772-9.

26. Szende A, Janssen B, Cabases J. Self-Reported Population Health: An International Perspective based on EQ-5D. Szende A, Janssen B, Cabases J, editors. Dordrecht: 
Springer Netherlands; 2014. Available from: http//link.springer.com/10.1007/97894-007-7596-1.

27. Whynes DK. Does the correspondence between EQ-5D health state description and VAS score vary by medical condition? Health Qual Life Outcomes. 2013;11:155.

28. Maatouk I, Wild B, Herzog W, Wesche D, Schellberg D, Schöttker B, et al. Longitudinal predictors of health-related quality of life in middle-aged and older adults with hypertension: results of a population-based study. J Hypertens. 2012;30(7):1364-72.

29. Huang IC, Frangakis C, Atkinson MJ, Willke RJ, Leite WL, Vogel WB, et al. Addressing ceiling effects in health status measures: a comparison of techniques applied to measures for people with HIV disease. Health Serv Res. 2008;43(1 Pt 1):327-39.

\section{Submit your next manuscript to BioMed Central and take full advantage of:}

- Convenient online submission

- Thorough peer review

- No space constraints or color figure charges

- Immediate publication on acceptance

- Inclusion in PubMed, CAS, Scopus and Google Scholar

- Research which is freely available for redistribution 